\title{
INFLUENCE OF ION CHANNEL ON ELECTRON BEAM PROPAGATION*
}

\author{
Han S. Uhm, Naval Surface Warfare Center, West Bethesda, MD 20817-5700 \\ E. H. Choi, J. J. Ko and H. M. Shin, Kwangwoon University, Seoul 139-701, Korea
}

The electron beam propagation from a gas-filled diode is investigated. The beginning portion of the electron beam pulse creates an ion channel not only inside the diode but also the region beyond the anode. A theoretical model is developed for a space-charge limited current of a relativistic electron beam propagating through an ion channel. A simple analytical expression of the space-charge limited current is obtained within the context of a thin beam approximation, where the conducting tube radius is much larger than the beam radius. Beam current propagating through an ion channel is measured experimentally for a mildly relativistic electron beam. Whenever the ion density inside a diode is the same as the beam electron density, the diode is short-circuited. The ion channel density at the short-circuiting time is numerically calculated and is used to estimate the space-charge limited current. It is shown that experimental data agree well with analytical results predicted by the theoretical model.

\section{INTRODUCTION}

There is a strong renewed interest ${ }^{1-6}$ in theoretical and experimental studies of the space-charge limited current for relativistic electron beams propagating through a grounded cylindrical conducting tube. As the charged beam current in a drift tube increases to a limiting value, electrostatic potential builds up due to the beam's self-space charge field and as a result, the beam cannot propagate, creating a virtual cathode in the drift tube. This limiting current is the ultimate restriction on power increase in various electron beam applications. Therefore, a correct estimation of the limiting current is one of the important tasks in these applications. The limiting current $\mathrm{I}_{\mathrm{L}}$ of a unneutral solid beam has been derived empirically for a uniform density and is expressed as ${ }^{1}$

$$
I_{L}=\frac{I_{A}\left(\gamma_{z}^{2 / 3}-1\right)^{3 / 2}}{1+2 \ln \left(R_{c} / R_{b}\right)},
$$

where $I_{A}=\mathrm{mc}^{3} / \mathrm{e}=17 \mathrm{kA}$ is the Alfven current, -e and $\mathrm{m}$ are charge and rest-mass of electrons, respectively, $\mathrm{c}$ is the speed of light in vacuum, $\gamma_{\mathrm{z}}$ is the relativistic mass ratio due to the axial motion of beam electrons, and $\mathrm{R}_{\mathrm{c}}$ and $\mathrm{R}_{\mathrm{b}}$ are radii of drift tube and beam, respectively. The limiting current in Eq. (1) represents an approximation that the beam is thin in comparison with the drift tube. We develop a theoretical model of the space-charge limited current for a relativistic electron beam propagation, including an ion channel, which will partially neutralize the space-charge

*This work was supported by IR Fund at NSWC and by KSEF field created by beam electrons.

\section{SPACE-CHARGE LIMITING CURRENT IN AN ION CHANNEL}

The relativistic mass ratio $\gamma$ associated with axial motion of electrons at the axis is expressed as

$$
\gamma=\gamma_{T}-\frac{I}{I_{A}}\left[1+2 \ln \left(\frac{R_{c}}{R_{b}}\right)\right] \frac{\gamma}{\sqrt{\gamma^{2}-1}},
$$

where the total mass ratio $\gamma_{\mathrm{T}}$ is defined by

$$
\gamma_{T}=\gamma_{z}+\delta \gamma=\gamma_{z}+\frac{\pi R_{b}^{2} n_{i} c e}{I_{A}}\left[1+2 \ln \left(\frac{R_{c}}{R_{b}}\right)\right]
$$

and use has been made of the definition $\beta=\left(\gamma^{2}-1\right)^{1 / 2} / \gamma$. Equation (1) is equivalently expressed as

$$
\frac{I}{I_{A}}=\frac{\left(\gamma_{T}-\gamma\right) \sqrt{\gamma^{2}-1} / \gamma}{1+2 \ln \left(R_{c} / R_{b}\right)}
$$

which has its maximum value at the relativistic mass ratio

$$
\gamma=\gamma_{T}^{1 / 3}
$$

Substituting Eq. (5) into Eq. (4), the space-charge limited current $\mathrm{I}_{\mathrm{L}}$ of a relativistic electron beam propagating through a ion channel is given by

$$
I_{L}=\frac{I_{A}\left(\gamma_{T}^{2 / 3}-1\right)^{3 / 2}}{1+2 \ln \left(R_{c} / R_{b}\right)}
$$

where $\mathrm{I}_{\mathrm{A}}=17 \mathrm{kA}$ is the Alfven current. Equation (6) with Eq. (3) is one of the main results of this article and can be used to investigate the space-charge limited current of an electron beam propagating through an ion channel. We remind the reader that the space-charge limited current in Eq. (6) recovers the previous result in Eq. (1) when the channel ion density approaches zero. The total relativistic mass ratio $\gamma_{T}$ in Eq. (3) is linearly increasing function of the ion density $n_{i}$. On the other hand, the limiting current $I_{L}$ in Eq. (6) is nonlinearly increasing function of the mass ratio $\gamma_{\mathrm{T}}$. Therefore, the limiting current is nonlinearly increasing 
function of the ion density when beam's axial motion is not ultra-relativistic. The limiting current in the ion channel increases drastically, whenever intensity of the ion channel increases.

\section{ELECTRON BEAM PROPAGATION THROUGH A GAS CELL}

An experiment has been carried out for a relativistic electron beam propagating through a gas-filled chamber with radius of $5 \mathrm{~cm}$. The electron beam is obtained from an accelerator which is a high-voltage Marx generator consisting of 12 capacitors. Each capacitor has $0.2 \mu \mathrm{F}$. The total stored energy of the accelerator with maximum charging voltage of $\pm 50 \mathrm{kV}$ can be raised to $3 \mathrm{~kJ}$. A relativistic electron beam with $600 \mathrm{kV}, 88 \mathrm{kA}$ and 60 nsec pulse length can be generated if the water-filled pulse forming line with characteristic impedance of $6.8 \Omega$ is impedance-matched to a field emission diode. However, in this experiment, we used the electron beams with $300 \mathrm{kV}$, $33 \mathrm{kA}$ in a gas-filled diode. The tungsten tip cathode in the diode has a 4-mm diameter and the stainless steel anode has a 20-mm aperture. The cathode and anode gap is kept to 3 $\mathrm{mm}$ throughout whole experiment. An aluminum foil with $15-\mu \mathrm{m}$ thickness is installed at $20-\mathrm{cm}$ downstream from the anode plane to separate the gas-filled region from propagation zone. More than 100 shots were made before replacing the aluminum foil. The diode pressure has been varied from 10 mTorr to 100 mTorr. The gas used in this experiment is air. To measure electron beam signals, a Faraday cup is located just behind the aluminum foil, where the pressure is kept to be $10^{-4}$ Torr. The electron beam current is measured by a Faraday cup. The B-dot probe at the diode chamber is used to measure diode current. The Cdot probe installed in the isolator section of the pulse forming line is used to measure diode voltage signal. These probes are connected to a four-channel digital oscilloscope with $2 \mathrm{GHz}$ sampling rate. Diagnostic coaxial cables are adjusted so that all the signals are triggered synchronously on the oscillograph.

Although the electron beam current inside the diode is more than $30 \mathrm{kA}$, the beam current beyond the anode is about $6 \mathrm{kA}$ or less due to a finite size of the anode aperture. Current measured at the Faraday cup is the beam current that propagates through the gas cell and penetrates through the aluminum foil. Stopping power of this foil is about $30 \mathrm{keV}$. The peak beam current has been measured in terms of the chamber pressure and is shown in Fig. 1. The closed dots represent experimental data and dotted curve is an approximate trace of the data. We believe that the majority of beam electrons may have much less than 300 $\mathrm{keV}$, which is the peak diode energy. The space-charge limited current associated with zero chamber pressure must be far less than the propagation currents shown in Fig. 1. Therefore, we believe that the beginning part of the beam pulse creates an ion channel through which the later portion of the beam pulse can propagate.

Ions are created from neutral particles by the electron impact ionization. The ion production rate $\mathrm{dn}_{\mathrm{i}} / \mathrm{dt}$ inside the diode by the electron impact ionization is given ${ }^{2,7}$

$$
\frac{d}{d t} n_{i}(t)=\frac{J_{d}(t) n_{g} \sigma(t)}{e}
$$

where $J_{d}(t)$ is the beam current density at the diode, $\sigma(t)$ is the ionization cross section and $\mathrm{n}_{\mathrm{g}}$ is the neutral number density. Secondary electrons, generated by the ionization, are assumed to instantaneously leave region of the diode electrons, being radially expelled by the large space-charge field of electrons accelerated by the diode voltage. Integrating Eq. (15) over time t, the ion density is expressed as

$$
n_{i}(t)=\frac{n_{g}}{e} \int_{0}^{t} d t^{\prime} J_{d}\left(t^{\prime}\right) \sigma\left(t^{\prime}\right)
$$

where the current density $J_{d}(t)$ in the gas-filled diode is given by

$$
J_{d}(t)=\frac{I_{d}(t)}{\pi R_{b}^{2}},
$$

and $I_{d}(t)$ is the diode current. The empirical cross section $\sigma(t)$ of neutral gases ${ }^{8}$ by the electron impact ionization is given by

$$
\sigma(t)=1.874 \times 10^{-24}\left(M^{2} x_{1}+C x_{2}\right) \quad\left(m^{2}\right),
$$

where $\mathrm{M}^{2}=3.83, \mathrm{C}=35.6$ for air, $\mathrm{x}_{1}=2 \beta(\mathrm{t})^{2} \ln \left[\gamma^{2}(\mathrm{t})-1\right]-1$, $\mathrm{x}_{2}=\beta(\mathrm{t})^{-2}$, and $\gamma(\mathrm{t})$ and $\beta(\mathrm{t}) \mathrm{c}$ are the relativistic mass ratio and velocity, respectively of beam electrons.



Fig. 1. Plot of the peak beam current propagated through gas cell versus the chamber pressure. Closed dots represents experimental data and dotted curve is an approximate trace of data.

A typical voltage pulse in the diode consists of three parts. They are the leading portion, main pulse and the tail. The leading portion, which is the first part of the diode 
pulse, is a low voltage portion accompanied by a small diode current. The main pulse, which is the $300 \mathrm{kV}$ and 30 kA current, is followed by a low-voltage, low-current tail. Typical experimental data indicates that the current levels of the leading portion and tail are one order in magnitude less than that of the main pulse. Therefore, the diode beam current $I_{d}(t)$ typically starts from a small beam current, increases to a peak value, and then decreases to a small tail current, as time goes by. Detailed features of the diode current must be experimentally obtained for individual pulse device, which has its unique machine and diode impedances. The ion density $\mathrm{n}_{\mathrm{i}}(\mathrm{t})$ inside the diode can be numerically calculated by substituting Eqs. (9) and (10) into Eq. (8) after digitizing experimental data of the diode beam current $\mathrm{I}_{d}(\mathrm{t})$ and voltage $V_{d}(t)$. Here the beam radius $R_{b}$ is assumed to be $1 \mathrm{~cm}$. The ion density $\mathrm{n}_{\mathrm{i}}(\mathrm{t})$ is numerically calculated as a function of time for various gas pressures.

It is required to consider the electron beam current inside a high-power diode with the presence of ions. Particularly when the ion density is the same as the beam electron density inside a diode, the diode may be shortcircuited. Since the cathode plasma can be considered as a metal surface whose work function is effectively zero, the cathode electron current supply is essentially unlimited. In this case the current that flows in the diode is determined by the modification of the equipotential contours in the diode due to the space charge of the electron current. Thus, the electron current in the diode must be the space-charge limited current, ${ }^{2}$ which originates from a zero electric field at the cathode even for a large voltage difference between the anode and cathode. The zero electric field at the cathode is the outcome of the potential modification due to the space-charge field of the negatively-charged electron current. The zero electric field regulates injection of electrons abundantly available at the cathode surface, essentially controlling the diode current. If the ion density inside the diode is the same as the beam electron density, there will be no space-charge field and no potential modification. The electric field at the cathode in this case will be a non-zero value, which forces all electrons at the cathode to flow to the anode instantaneously, shortcircuiting the diode. Thus, the ion density increases from zero to the value of the beam electron density as time goes by. When the ion density is the same as the beam electron density, the voltage and current pulses drop to zero and remain to be zero after this time. Therefore, the highest ion density attainable is the ion density the same as the beam electron density at the time. The ion density in a low pressure diode builds up very slowly for the pressure of 10 mTorr. The ion density in this low pressure diode increases to be the same as the beam electron density in the tail portion of the beam pulse, where the diode beam current is low. The ion density increases more rapidly as the diode pressure increases. This means that the time at which the ion density is the same as the beam electron density occurs at the earlier portion of the beam, where the diode beam current and beam electron density are high. The peak ion density at a certain optimum pressure will coincide the highest beam electron density corresponding to the peak value of diode current. The ion density in a very high pressure diode builds up too quickly and reaches the same value of the beam electron density at the beginning portion of the diode pulse. The highest ion density attainable in this high pressure diode is therefore low. We summarize that the maximum ion density inside the diode increases from a small value to a peak and then decreases to a small value again, as the diode pressure increases from $10 \mathrm{mTorr}$ to 100 mTorr.

Figure 2 shows the ion density $n_{i}$ inside the diode at the diode shorting time, when the ion density is the same as the beam electron density. In obtaining the numerical results in Fig. 5, we have used the digitizing experimental data of the diode beam current $\mathrm{I}_{\mathrm{d}}(\mathrm{t})$. As expected, the ion density in Fig. 2 increases to a peak value and decreases, as the diode pressure increases from 20 mTorr to 60 mTorr.

However, this numerical calculation indicates more diversified detailed structures than the general description mentioned above. These minor structures may be caused by the ion density calculation including the highlyfluctuating experimental data of the beam current. We found that the profile of the ion density $\mathrm{n}_{\mathrm{i}}$ at the diode shorting time in Fig. 2 is very similar to that of peak propagation current in Fig. 1 measured by the Faraday cup.

However, we remind the reader that the peak propagation current in Fig. 1 is enhanced considerably more than the peak ion density in Fig. 2.

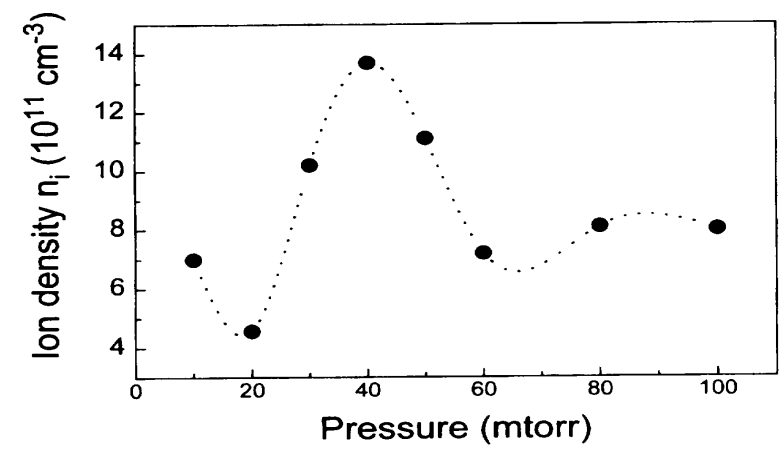

Fig. 2. Plot of ion density at the time of peak current propagation versus the chamber pressure obtained numerically from Eq. (8).

\section{REFERENCES}

[1] L.S. Bogdankevich and A. A. Rukhadze, Sov. Phys. Usp. 14, 163 (1971).

[2] R.B. Miller, An Introduction to the physics of Intense Charged Particle Beam (Plenum, New York, 1982), Chaps.2, 3 and 5.

[3] R.C. Davidson, Physics of Nonneutral Plasmas (AddisonWesley, Reading, MA, 1990), Chap. 9.

[4] C. Grebogi and H. S. Uhm, Phys. Fluids 31, 1277 (1988).

[5] M. Reiser, Phys. Fluids 20, 477 (1977).

[6] H.S. Uhm, Phys. Fluids B 5, 1919 (1993).

[7] C.L. Olson, Phys. Rev. A 11, 288 (1975).

[8] F.F. Rieke, W. Prepejchal, Phys. Rev. A. 6, 1507 (1972). 